
artículo analiza una serie de datos empíricos -críticas de traducciones literarias y premios nacionales a la traducción- y testimonios (entrevistas con traductoras) para plantear las razones sociales y profesionales de esa invisibilidad y falta de reconocimiento.

PALABRAS CLAVE: Traducción literaria, traducción profesional, sociología aplicada a la traducción, Pierre Bourdieu, traductoras.

\title{
De la profesionalización a la invisibilidad: las mujeres en el sector de la traducción editorial
}

FruELA FERNÁNDEZ

Universidad de Granada
From professionalism to invisibility: women translators in the publishing field

Professional translation in Spain is an increasingly feminized sector. Nevertheless, this situation of the labour market is not producing a greater visibility of women translators. Employing Pierre Bourdieu's sociological framework, this paper analyzes a set of data-reviews of literary translations and national awards to translation- and reflections (interviews with women translators) in order to consider social and professional causes for this lack of visibility and acknowledgement.

KEY WORDS: Literary translation, professional translation, Sociology applied to Translation, Pierre Bourdieu, women translators. 


\section{INTRODUCCIÓN: LA PERSISTENCIA INCONSCIENTE DE UNA CONSTRUCCIÓN SOCIAL}

50 Considerada históricamente como una labor creativa «inferior», la traducción ha servido también como vía de acceso femenino al ámbito literario y profesional, dentro de una estructura social que relegaba a las mujeres a tareas consideradas de menor importancia. Esta restricción social ha tenido una larga influencia histórica que, sin duda, ha condicionado tanto el estatuto de la profesión como el de las profesionales. Desde este planteamiento y apoyándose en diversos trabajos sociológicos - especialmente aquellos influidos por Pierre Bourdieu一, este artículo analizará el ámbito de la traducción literaria en España para plantear, de modo empírico, si aún se manifiesta una relación social entre la consideración social del «traductor» - como grupo inferior en la jerarquía de los campos literarios (cf. Fernández, 2oria) - y la constante feminización de este ámbito.

Para ello se recurrirá al análisis de dos tipos de materiales que permiten observar esa presencia y repercusión: en primer lugar, un corpus de reseñas de traducciones, publicadas en la prensa española (I999-2008); en segundo lugar, la historia de los premios nacionales de traducción (1984-2009). Asimismo, estos datos serán contextualizados recurriendo a las declaraciones de distintas traductoras profesionales, recabadas por el autor en 2010 mediante breves cuestionarios informales. De este modo, se intentará analizar hasta qué punto el ámbito de la traducción editorial refleja aún la asociación histórica entre ambos grupos «dominados»: mujeres y traductores.

\section{LA CONSTRUCCIÓN SOCIAL COMO CONDICIONANTE LABORAL}

Una de las premisas de la sociología crítica es cuestionar aquellas construcciones sociales que se legitiman y presentan como "hechos naturales», que los propios sujetos llegan con frecuencia a asumir en su vida cotidiana. Uno de los paradigmas de esta construcción social naturalizada es aquel que concierne a la diferencia «hombre-mujer», a través de todas las características atribuidas a cada sexo e integradas, a su vez, en un conjunto de oposiciones más amplio que hace de la realidad social una estructura de connotaciones, a un mismo tiempo, sexuales y sociales (Bourdieu, I998: 17-26).

Entre las múltiples consecuencias de esta construcción sexual de la realidad, aquella que afecta al ámbito laboral es una de las principales, ya que la catalogación de actividades consideradas «propias» e «impropias» de cada sexo influye decisivamente en el acceso al trabajo: las profesiones tienden a considerarse "masculinas» o «femeninas» (Anker, I998: 250-296) de acuerdo con las características atribuidas socialmente a cada sexo. De este modo, se produce una segregación horizontal y vertical del mercado de trabajo (Ibáñez Pascual, 2008, 20Io): las mujeres no sólo tienden a concentrarse en profesiones «femeninas», sino que también suelen predominar en los puestos de menor responsabilidad e ingresos. Asimismo, la segregación tiende a ser un elemento estructural y estructurante del mercado (Ibáñez Pascual, 2010): a la vez que los contenidos de las ocupaciones suelen estar asociados a los estereotipos sexuales (Anker, I998), la tendencia condiciona las alternativas futuras, en la medida en que la elección ocupacional es emulativa y las personas jóvenes tienden a elegir estudios donde haya referentes de su propio 
sexo (Cockburn, 1987; Ibáñez Pascual, 2010)․ En el caso concreto de España (Ibáñez Pascual, 2008), el 6r\% de los trabajadores de uno y otro sexo se encuentran en ocupaciones mayoritariamente masculinas o mayoritariamente femeninas; asimismo, la segregación ocupacional no se ha reducido con la mayor participación laboral de la mujer, sino que ha aumentado ligeramente desde la década de 1980 .

En esta estructura de connotaciones sociales, las profesiones artísticas constituyen un ámbito de segregación notable, pues, si bien las mujeres predominan entre los «amateurs», entre los practicantes no profesionales de las artes, su acceso al estatuto profesional se revela de especial dificultad (Buscatto, 2007; Naudier, 2007; Trasforini, 2007; Cacouault-Bitaud \& Ravet, 2008; Bielby, 2009). En este contexto, la traducción ha constituido a lo largo de la historia una labor de considerable repercusión en la vida intelectual de las mujeres; su estatuto de tarea considerada "poco creativa», «dominada» frente a la escritura, ha servido como modo de acceso indirecto de la mujer al campo literario (Sirois, I997; Delisle, 2002; Pieretti, 2002; Agorni, 2005; Matter-Seibel, 2006; Kalinowski, 2007). La concepción de la traducción como labor pasiva, reproductiva, opuesta a la escritura como producción o generación activa (Chamberlain, I988) la volvía socialmente apta para las mujeres; esta discriminación, sin embargo, fue aprovechada por distintas traductoras para convertir la traducción en una herramienta que les permitía, por un lado, la adquisición de un capital literario que les estaba vetado y, por otro, la introducción de ideas que no hubiesen sido

I En términos antropológicos, esta condición emulativa puede relacionarse con la formación del «habitus» de cada sujeto (Bourdieu, I980: 108): la estructura social de posibilidades que encuentran los distintos sujetos define las expectativas en torno aquello que considerarán «posible» o «imposible». aceptadas en una obra «original». La traducción, de este modo, se ha configurado de acuerdo con la estructura de dominación social: su estatuto de tarea «secundaria» en la sociedad la volvía afín a un grupo considerado «secundario» ${ }^{2}$.

En esta línea, los datos estadísticos recientes demuestran que la traducción es en España una tarea profesional en proceso continuo de feminización, como se observa tomando los datos del primer y segundo Libro Blanco de la Traducción de ACEtt (Macías Sistiaga, Fernández Cid \& Martín Caño, 1997; Marinas, 20ro). En 1997, fecha del primer estudio, el número de hombres dedicados a la traducción de libros aún era superior al de mujeres $(52,6 \%)$, aunque esta preponderancia se demuestra relativa al plantearla con otros datos: en primer lugar, de acuerdo con el censo del Instituto Nacional de Estadística, las mujeres suponían en aquel momento el $35,05 \%$ del mercado laboral frente al $64,95 \%$ masculino; por otro lado, de las mujeres dedicadas a la traducción de libros, el $49 \%$ se dedicaba en exclusiva a esta tarea frente al $37 \%$ de los hombres. En consecuencia, ya en ese momento la profesión mostraba un sesgo femenino, puesto que el porcentaje de mujeres en el sector superaba ampliamente su inserción en el conjunto del mercado laboral ( $47,4 \%$ frente a $35,05 \%$ ) y su dedicación laboral era mayor que la de sus compañeros varones. En 2009, fecha del segundo estudio, la tendencia se mantenía: las mujeres, aunque suponen el 44,07\% del mercado laboral conjunto, constituyen un $54, \mathrm{r} \%$ del colectivo de traductores de libros y su dedicación sigue aumentando, ya que el $57,8 \%$ de ellas tienen la traducción como labor profe-

2 La condición ancilar de la traducción hallaría su paradigma en aquellas traductoras que fueron amantes o esposas de autores más célebres y que, en ocasiones, ejercieron su tarea para favorecer la carrera intelectual de su pareja (Sirois, I997: 105-I09) 
sional exclusiva frente al 39,7\% de los hombres. En la actualidad, por tanto, la traducción de libros continúa la tendencia social e histórica y 52 se muestra como un sector laboral feminizado, ya que las mujeres se orientan a él antes que a otros -como demuestra su mayor presencia frente al conjunto del mercado- y con mayor dedicación, frente a la tendencia de los hombres a simultanear varias profesiones.

\section{LA VISIBILIDAD: TRADUCCIÓN Y SUPLEMENTOS CULTURALES}

La pregunta que, desde una sociología crítica, se impone a la vista de estos datos estadísticos es relativamente clara: una mayor presencia de las mujeres en el sector editorial ¿se manifiesta en una mayor visibilidad? Por intuición, la respuesta es probable que sea negativa; es necesario, sin embargo, acercarse a los planteamientos empíricos para comprobar si la intuición es cierta o errada.

En primer lugar, esta investigación se centrará en el ámbito de la crítica literaria de traducciones. En tanto que instrumento de la cultura «legítima», la crítica suele ser representativa de las concepciones que prevalecen en ese ámbito «legítimo» e «institucional» y permite, por tanto, observar el funcionamiento soterrado, «inconsciente» de este ámbito. Para ello se recurrirá a los datos de una investigación previa (Fernández, 2orrb), centrada en el ámbito de la crítica de traducciones literarias en prensa y desarrollada a partir del análisis de tales críticas y reseñas publicadas durante una década (1999-2008) en los cuatro diarios generalistas españoles de mayor tirada (El País, El Mundo, ABC y La Vanguardia). En términos generales, este análisis había revelado un notable desinterés de la crítica respecto a la «calidad» de las traducciones, ya que sólo un II,42\% (I846) de las

Tabla i. Valoraciones de calidad CLASIFICADAS SEGÚN EL SEXO DEL TRADUCTOR

Sexo traductor

\begin{tabular}{ccccc}
\hline Valoraciones & $H$ & $M$ & $E q^{*}$ & Total \\
\hline Número & I327 & 430 & 89 & 1846 \\
\hline Porcentaje & 71,86 & 23,29 & 4,82 & I00 \\
\hline
\end{tabular}

*Eq: Equipo mixto de traductores, formado por hombre(s) y mujer(es)

críticas publicadas en prensa durante este periodo incluían algún tipo de valoración de calidad. A pesar de esta tendencia, el axioma de trabajo que plantearían los datos «brutos» resulta relativamente claro: puesto que, durante el periodo estudiado, las mujeres han ido aumentando su presencia en la profesión y se han dedicado a ella de modo más exclusivo que los hombres, las valoraciones de calidad deberían ser semejantes en número para aquellas traducciones realizadas por hombres y aquellas realizadas por mujeres.

Sin embargo, al desglosar los datos empiezan a observarse marcadas desviaciones respecto al equilibrio aparentemente «lógico», que pueden como indicadores de la configuración del campo: clasificando las valoraciones de calidad de acuerdo con el sexo de la persona que ha realizado la traducción (tabla I), se observa, en primer lugar, que las traducciones realizadas por hombres obtienen el 7r,86\% de las valoraciones (tanto positivas como negativas), mientras que las traducciones hechas por mujeres suponen tan sólo un 23,29\% y que el resto de valoraciones se otorgan a los textos traducidos por equipos de trabajo mixtos de hombre(s) y mujer(es). Pese a todo, esta menor presencia femenina no se debe a una posible percepción marcadamente negativa de su trabajo, ya que tanto traductores como traductoras tienden a recibir más valoraciones positivas que negativas (tabla 2); 
TABla 2. VAloraciones de CALIDAD

DE LAS TRADUCCIONES CLASIFICADAS SEGÚN EL SIGNO DE LA VALORACIÓN Y EL SEXO DE LOS TRADUCTORES

Sexo traductor

\begin{tabular}{ccccc}
\hline Valoración & $H$ & $M$ & $E q$ & Total \\
\hline Muy Positiva & 650 & I94 & $4 \mathrm{I}$ & 885 \\
\hline Positiva & 453 & I26 & 34 & 613 \\
\hline Neutral & 70 & 22 & 4 & 96 \\
\hline Negativa & I23 & 65 & 8 & 196 \\
\hline Muy Negativa & 31 & 23 & 2 & 56 \\
\hline Total & I327 & 430 & 89 & I846
\end{tabular}

pese a todo, también en este punto los hombres sobrepasan a las mujeres: las valoraciones "positivas» $\mathrm{y}$ «muy positivas» suponen un $83, \mathrm{I} 2 \%$ de las valoraciones efectuadas sobre traducciones hechas por hombres, frente al $74,42 \%$ de las traducciones hechas por mujeres.

Estos primeros datos ya permiten formular algunas hipótesis: dado que las mujeres suponen una parte mayor y de mayor producción (cuantitativa) de la profesión y que sus traducciones, en términos generales, muestran una tendencia a ser valoradas de manera casi tan positiva como aquellas realizadas por hombres (tabla 2), la razón de que sus traducciones reciban un porcentaje global de valoraciones tan bajo (tabla I) no debe explicarse por una cuestión de «calidad», sino a partir de una configuración previa del mercado laboral. En primer lugar, la crítica, como instrumento de la cultura «legítima», tiende a centrarse en obras que se inserten en esta categoría «legítima» y constituye, por tanto, un indicador de la existencia, aparición o consolidación de tales géneros; como se observará más adelante, las valoraciones de calidad de la traducción demuestran esta tendencia al concentrarse en las obras de géneros más «formales», más «legítimos», en suma, «más literarios». Se puede plantear la hipótesis, en consecuencia, de que la baja representación de las mujeres se debería al tipo o género de obras que traducirían: frente a los hombres, dedicados a la traducción de aquellas obras consideradas «legítimas» (cf. Kalinowski, 2002), las mujeres se centrarían en textos considerados «menos cultos» -en especial aquellos del sector de gran producción-, que no serían tratados por la crítica o en los que la traducción no se consideraría un factor importante. Por otra parte, la baja remuneración de la profesión de traductor en España (cf. Macías Sistiaga, Fernández Cid \& Martín Caño, 1997; Fernández, 2oro; Marinas, 2010) constituye un factor estructural que, con gran probabilidad, dificulta el criterio de selección de los profesionales, entre los que las mujeres son mayoría: la restricción económica fomenta la diversidad de encargos y tiende a reducir las obras consideradas «legítimas», de mayor dificultad y con menor peso en el mercado. Asimismo, la mayor carga familiar femenina (Papí \& Frau, 2005) podría constituir un factor que limitase también la posibilidad de elección; en un conjunto de encuestas paralelas a este trabajo, dos traductoras - con una experiencia profesional de 25 años, la primera, y 50 la segunda - se referían también a esta posibilidad:

Si pienso en mi carrera, por ejemplo, durante unos cuantos años (pocos) he preferido tener traducciones fáciles porque bastante lío tenía con mis niñas pequeñas. Y puedo concebir que esa actitud en muchas mujeres se prolongue durante mucho tiempo. (Traductora 2)

Otra cosa es que, más tarde, [las mujeres] logren situarse profesionalmente por encima de los hombres. Creo que aquí juega un papel 
Tabla 3. Valoraciones de Calidad

CLASIFICADAS SEGÚN EL GÉNERO DE LA OBRA Y EL SEXO DEL AUTOR DE LA TRADUCCIÓN

\begin{tabular}{ccccc}
\hline & \multicolumn{4}{c}{ Sexo traductor } \\
\hline Género & $H$ & $M$ & $E q$ & Total \\
\hline Narrativa & 443 & 215 & 24 & 682 \\
\hline Poesía & 543 & 99 & 38 & 680 \\
\hline Ensayo & 318 & I05 & 26 & 449 \\
\hline Otros & 23 & II & I & 35 \\
\hline Total & I327 & 430 & 89 & I846
\end{tabular}

importante la tradición, que margina a la mujer, y los condicionantes que le impone la maternidad. (Traductora 4)

Esta hipótesis en torno a la importancia de la dedicación profesional puede precisarse si se estudia, por un lado, la distribución sexual según géneros literarios y, por otro, el estatuto profesional de ambos grupos. El primer postulado (tabla 3) muestra algunos detalles relevantes: aunque en todos los géneros textuales tienen los traductores mayor presencia que las traductoras y que los equipos mixtos, su presencia es más baja en la narrativa (64,9\% de las valoraciones, tanto positivas como negativas), aumenta en el ensayo (70,8\%) y alcanza su punto superior en la poesía (79,85\%). Esta polarización parece confirmar lo ya planteado: los traductores tienden a orientarse hacia los géneros de mayor capital simbólico, aquellos más «formales» y «nobles», mientras que las traductoras hallan más espacio entre los géneros de mayor rendimiento comercial y, por lo general, menos legitimados literariamente. Una traductora con 30 años de experiencia profesional en el sector del libro definía su experiencia de un modo semejante:

Tabla 4. Valoraciones de calidad CLASIFICADAS SEGÚN PROFESIÓN Y SEXO DEL AUTOR DE LA TRADUCCIÓN

\begin{tabular}{lcccc}
\hline & \multicolumn{4}{c}{ Sexo traductor } \\
\hline Clas trad & $H$ & $M$ & $E q$ & Total \\
\hline Traductor & 357 & I99 & 32 & 588 \\
\hline Traductor vet. & 289 & IO0 & I2 & 4 OI \\
\hline Poeta & 248 & 30 & I3 & 29I \\
\hline Escritor & II7 & 33 & 2 & I52 \\
\hline Profesor Univ. & II7 & 26 & 5 & I48 \\
\hline Especialista & 78 & 36 & 22 & I36 \\
\hline Poeta-traductor & 64 & I & I & 66 \\
\hline Ensayista & 2 I & I & $\circ$ & 22 \\
\hline Escritor-traductor & I8 & $\circ$ & $\circ$ & I8 \\
\hline Editor & 5 & 4 & 2 & II \\
\hline Filósofo & II & $\circ$ & $\circ$ & II \\
\hline Profesor & 2 & $\circ$ & $\circ$ & 2 \\
\hline Total & I327 & 430 & 89 & I846 \\
\hline
\end{tabular}

A mí me parece que a las mujeres se les encargan menos traducciones de obras «sesudas», de pensamiento filosófico. No sé si se debe a que por tradición los editores confían más en los traductores hombres o al hecho de que a las mujeres se nos ha relegado a saberes de cualquier tipo menos los relacionados con la elaboración y creación del pensamiento... Parecería que las mujeres estamos más dotadas para las obras con carga sentimental, evocadora, romántica... Pero no me extrañaría nada que hubiera más traductores de poesía que traductoras... (Traductora 6 )

En esta oposición, pese a todo, conviene notar cómo ciertas características consideradas 
TABla 5. VAloraciones de CAlidad

CLASIFICADAS SEGÚN EL ESTATUTO

PROFESIONAL DEL AUTOR DE LA TRADUCCIÓN (HOMBRES)

\begin{tabular}{lc}
\hline Estatuto prof. & N. ${ }^{\circ}$ valoraciones \\
\hline Traductor & 357 \\
\hline Traductor veterano & 289 \\
\hline Poeta & 248 \\
\hline Escritor & II7 \\
\hline Profesor universitario & II7 \\
\hline Especialista & 78 \\
\hline Poeta-Traductor & 64 \\
\hline Ensayista & $2 \mathrm{I}$ \\
\hline Escritor-Traductor & I8 \\
\hline Filósofo & II \\
\hline Editor & 5 \\
\hline Profesor & 2 \\
\hline Total & I327 \\
\hline
\end{tabular}

«femeninas» - y vistas como «innatas» en las mujeres- pueden ser un signo de distinción en los varones del campo artístico, como ocurre con la «sensibilidad» o la «emotividad» (cf. Albenga, 2007; Naudier, 20I0); en cambio, las características «masculinas», como la «erudición», pueden considerarse negativas cuando se observan en una mujer, como se manifestaba en la conocida mofa de Kant en su tratado sobre «lo bello» y «lo sublime»: «A una mujer con la cabeza llena de griego, como la señora Dacier [traductora de la Ilíada], o que sostiene sobre mecánica discusiones fundamentales, como la marquesa de Châtelet [matemática, traductora de Newton y pareja de Voltaire], parece que no
TABla 6. VALORACIONES DE CALIDAD CLASIFICADAS SEGÚN EL ESTATUTO PROFESIONAL DEL AUTOR DE LA TRADUCCIÓN (MUJERES)

\begin{tabular}{lc}
\hline Estatuto prof. & N. ${ }^{\circ}$ valoraciones \\
\hline Traductora & I99 \\
\hline Traductora veterana & I00 \\
\hline Poeta & 30 \\
\hline Escritora & 33 \\
\hline Profesora universitaria & 26 \\
\hline Especialista & 36 \\
\hline Poeta-traductora & $\mathrm{I}$ \\
\hline Ensayista & $\mathrm{I}$ \\
\hline Editora & 4 \\
\hline Total & 430 \\
\hline
\end{tabular}

le hace falta más que una buena barba» (Kant, I764 [1978]: I48).

Al considerar las valoraciones de calidad según los estatutos profesionales-intelectuales en su conjunto (tabla 4) y desglosándolos después por cada sexo (tablas 5 y 6) ${ }^{3}$, se observa una segunda polarización relevante: aunque en ambos grupos son los traductores principiantes

3 Para la creación de las categorías empleadas, se procedió a un cotejo de materiales diversos: currículos de los sujetos, catálogos de publicaciones (ISBN para obras publicadas y Dialnet para la producción científica) y otros factores de consideración social, que pudieran matizar los datos brutos (premios, homenajes, reconocimientos, menciones en artículos de las publicaciones analizadas). Si bien en algunos casos la categorización apenas planteó dificultades, en otros casos se ha recurrido a categorías duales («escritortraductor», «poeta-profesor», etc.) o incluso a una categoría ambigua («especialista», que engloba a algunos sujetos con diversas posiciones dentro de un mismo ámbito de conocimiento, sin que ninguna de ellas resulte predominante) para reflejar la diversidad del mundo intelectual. 
TAbla 7. VAloraciones de CALIDAd Clasificadas SEgún la lengua original de la obra Y EL SEXO DEL AUTOR DE LA TRADUCCIÓN

Sexo traductor

\begin{tabular}{lccc}
\hline Lengua or. & $H$ & $M$ & $E Q$ \\
\hline Inglés & 473 & I40 & $3 \mathrm{I}$ \\
\hline Alemán & 234 & 72 & 7 \\
\hline Francés & 204 & 74 & $\mathrm{I} 6$ \\
\hline Italiano & 83 & $\mathrm{I} 7$ & 2 \\
\hline Portugués & 56 & $\mathrm{IO}$ & $\mathrm{I}$ \\
\hline Catalán & 45 & $\mathrm{I} 3$ & $\mathrm{I}$ \\
\hline Latín & 36 & 6 & 2 \\
\hline Ruso & 24 & $\mathrm{I} 5$ & 6 \\
\hline Griego Clásico & $2 \mathrm{I}$ & 6 & 2 \\
\hline Chino & 20 & $\mathrm{II}$ & $\mathrm{I}$ \\
\hline Varias & $\mathrm{I} 9$ & $\mathrm{I}$ & $\mathrm{O}$ \\
\hline Árabe & $\mathrm{I} 6$ & $\mathrm{II}$ & 2 \\
\hline Japonés & $\mathrm{I} 6$ & 8 & 2 \\
\hline Griego Moderno & 9 & 7 & $\mathrm{I}$ \\
\hline Polaco & 8 & 4 & $\mathrm{I}$ \\
\hline Sueco & 8 & $\mathrm{I}$ & $\mathrm{O}$ \\
\hline Gallego & 7 & 5 & $\mathrm{O}$ \\
\hline Persa & 5 & $\mathrm{I}$ & 2 \\
\hline Danés & 5 & $\mathrm{I}$ & $\mathrm{I}$ \\
\hline Rumano & 5 & $\mathrm{O}$ & $\mathrm{I}$ \\
\hline Hebreo & 4 & 4 & $\mathrm{O}$ \\
\hline Albanés & 4 & $\mathrm{O}$ & $\mathrm{O}$ \\
\hline Sánscrito & 3 & 2 & $\mathrm{I}$ \\
\hline & & &
\end{tabular}

$\mathrm{y}$ veteranos quienes reciben mayor número de valoraciones, las «traductoras» (tanto de una categoría como de otra) suponen una parte muy superior dentro del conjunto de mujeres $(69,5 \%)$ que los «traductores» en el conjunto de hombres (48,7\%); de modo inverso, los «escritores» pesan más entre las traducciones hechas por hombres $(35,27 \%)$ que las «escritoras» entre las mujeres $(\mathrm{I} 5,8 \%)$. Si se consideran las valoraciones de calidad como un modo de «corte» en la estructura

\begin{tabular}{|c|c|c|c|}
\hline & \multicolumn{3}{|c|}{ Sexo traductor } \\
\hline Lengua or. & $H$ & $M$ & $E Q$ \\
\hline Islandés & 3 & o & o \\
\hline Húngaro & 2 & 2 & 0 \\
\hline Provenzal & 2 & I & o \\
\hline Gaélico & 2 & I & o \\
\hline Turco & 2 & 0 & o \\
\hline Vasco & 2 & 0 & I \\
\hline Pali & 2 & o & $\mathrm{o}$ \\
\hline Checo & $\mathrm{I}$ & 4 & $\mathrm{I}$ \\
\hline Noruego & I & 2 & o \\
\hline Holandés & I & I & o \\
\hline Asturiano & I & I & o \\
\hline Yidis & I & o & 3 \\
\hline Acadio & I & o & $\mathrm{O}$ \\
\hline Castellano & I & o & o \\
\hline Occitano & I & o & o \\
\hline Celta & I & o & o \\
\hline Serbio & o & 2 & O \\
\hline Indonesio & o & 2 & o \\
\hline Anglosajón & o & I & o \\
\hline Arameo & o & I & O \\
\hline Coreano & o & I & I \\
\hline Croata & $\mathrm{O}$ & I & 2 \\
\hline Spanglish & o & I & $\mathrm{O}$ \\
\hline Finlandés & o & o & I \\
\hline
\end{tabular}

del campo literario-editorial, se puede sugerir que las traductoras no sólo tienden a concentrarse en géneros considerados «menos legítimos», sino que tendrían una mayor implicación profesional y, por lo general, no compaginarían la traducción con otras tareas creativas del campo literario; en cambio, los hombres no sólo se dedicarían a géneros más valorados, sino que, con más frecuencia que las mujeres, ejercerían la traducción como complemento - económico 
o simbólico- a una posición de "creación» dentro del campo literario. Asimismo, como se ha indicado en otros estudios (Fernández, 20IIa y 2orrb), la «multiposicionalidad», es decir, la compaginación de distintas tareas dentro del campo literario, tiende a ser un factor que otorga mayor «fiabilidad», mayor «confianza» en la calidad de la traducción, con independencia de sus características comprobables.

Un último factor de relevancia para el estudio de las distinciones podría hallarse en el estudio de las lenguas donde predominan traductores y traductoras (tabla 7), ya que, como se ha planteado, tanto el capital como la especialización de cada una varía. Al analizar esta estructura, se observa cómo los hombres dominan las lenguas «centrales» del sistema internacional (inglés, francés, alemán, italiano; cf. Heilbron 1999 y 2000) mientras que las mujeres se hacen más presentes a medida que la lengua pierde centralidad: su presencia en los idiomas «semiperiféricos» (ruso, chino, árabe, japonés) se acerca a la de los hombres y llega a veces a destacar en las lenguas «periféricas» (gallego, hebreo, indonesio, checo, húngaro); por otra parte, la presencia masculina es por completo dominante en las lenguas clásicas - latín y griego-, de escaso capital comercial, pero alto capital literario y universitario. Esta configuración de los idiomas evidenciaría que las lenguas periféricas constituyen una oportunidad comercial y cultural para las traductoras, ya que, frente a lenguas de mayor capital dominadas por traductores y profesores, el carácter marginal de estas lenguas ofrecería «nichos de mercado» que facilitan el acceso a una posición de especialista. Asimismo, este dato permitiría plantear la posibilidad de una cierta homología entre el espacio de las reseñistas y aquel de las traductoras; como se ha planteado (Fernández, 2orrb), las colaboradoras de los suplementos, al igual que parece ocurrir con las traductoras profesionales, tienden a concentrarse en reseñas de narrativa reciente - en especial aquella más cercana a la «gran producción»- $\mathrm{y}$ en otras obras consideradas «menos legítimas» (autoayuda, ensayo divulgativo). Sin embargo, diversas críticas que han alcanzado una posición relevante en el espacio de los suplementos también presentan una trayectoria de especialización en lenguas periféricas: Clara Janés (El Cultural, traductora del checo, Premio Nacional de Traducción), Chantal Maillard ( $\mathrm{BCD}$ y Babelia, especialista en sánscrito y cultura india) o Anne-Hélène Suárez (Babelia, sinóloga).

\section{EL RECONOCIMIENTO: PREMIOS INSTITUCIONALES A LA TRADUCCIÓN}

El «inconsciente» de la cultura «legítima», sus estructuras y configuraciones sociales, pueden revelarse de manera muy notable a través del análisis de los reconocimientos institucionales, como los premios. Por lo tanto, para mesurar la visibilidad de las mujeres en el ámbito de la traducción literaria resulta indispensable analizar los premios a la traducción concedidos por el Ministerio de Cultura español. Los dos premios existentes en esta categoría se construyen por analogía con otros premios de las Letras: por un lado, un premio a una obra concreta, el Premio Nacional a la Mejor Traducción, que surge en I984 a partir del antiguo Premio Fray Luis de León; por otro, el premio a una trayectoria, el Premio Nacional a la Obra de un Traductor, creado en 1989 .

Ahora bien, antes de pasar al análisis de la configuración por sexos resulta importante recordar la configuración profesional-intelectual a la que se hizo referencia en el epígrafe previo. Un axioma central de la sociología crítica es que el «gusto» está condicionado por el 
Tabla 8. Premios Nacionales a la Mejor

Traducción (I984-2009) Desglos ados SEGÚN LA CATEGORÍA PROFESIONAL Y EL SEXO

DEL PREMIADO

\begin{tabular}{|c|c|c|c|c|}
\hline \multirow[b]{2}{*}{ Estatuto prof. } & \multicolumn{4}{|c|}{ Sexo traductor } \\
\hline & $H$ & $M$ & $E q$ & Total \\
\hline Profesor universitario & $\mathrm{I} 2$ & 2 & O & I4 \\
\hline Traductor veterano & 6 & I & I & 8 \\
\hline Poeta & 2 & o & o & 2 \\
\hline Poeta-profesor & 2 & 0 & o & 2 \\
\hline Traductor-profesor & I & I & o & 2 \\
\hline Escritor & I & o & o & I \\
\hline Poeta-traductor & I & $\mathrm{O}$ & O & $\mathrm{I}$ \\
\hline Teólogo & $\mathrm{I}$ & $\mathrm{O}$ & O & I \\
\hline Traductor-escritor & I & $\mathrm{O}$ & $\mathrm{O}$ & I \\
\hline Total & 27 & 4 & $\mathrm{I}$ & 32 \\
\hline
\end{tabular}

«habitus», es decir, por la formación y la trayectoria social (Bourdieu, 1979); esto implica, por lo tanto, que la influencia de cada tipo de "gusto» variará según el ámbito analizado y según los grupos situados en posiciones dominantes. Una revisión sistemática del Boletín Oficial del Estado para conocer los procedimientos de constitución de los jurados permite observar la existencia de una parte fija - cuatro miembros de las Academias de la Lengua u órganos homólogos (Real Academia Española, Real Academia Gallega, Real Academia de la Lengua Vasca e Instituto de Estudios Catalanes), un número variable de representantes de asociaciones de traductores (ACEtt y/o APETI) y los autores premiados en la edición previa- y de otra sección del jurado cuya procedencia varía según los años, pudiendo incluir tanto a representantes de otros organismos (Escuela de Traductores de Toledo, Conferencia de Rectores de las Universidades Españolas, Federación de Asociaciones de Periodistas de España) como a
Tabla 9. Premios Nacionales a la Mejor Traducción (1984-2009) Desglosados SEGÚN EL GÉNERO DE LA OBRA TRADUCIDA Y EL SEXO DEL PREMIADO

\begin{tabular}{lcccc}
\hline & \multicolumn{4}{c}{ Sexo traductor } \\
\hline Género & $H$ & $M$ & $E q$ & Total \\
\hline Poesía & I5 & $\circ$ & $\circ$ & I5 \\
\hline Narración & 7 & 3 & I & II \\
\hline Religión & 3 & $\circ$ & $\circ$ & 3 \\
\hline Filosofía & I & I & $\circ$ & 2 \\
\hline Teatro & I & $\circ$ & $\circ$ & I \\
\hline Total & 27 & 4 & I & 32 \\
\hline
\end{tabular}

especialistas designados por el propio Ministerio. Los jurados, en consecuencia, se constituyen de un modo heterogéneo, pero sus miembros tienden a polarizarse entre dos grupos: profesores de universidad —en especial filólogos- y traductores de literatura con una trayectoria representativa. Considerando esta disposición profesional y analizando la historia de ambos premios (cf. tablas 8 y 9), puede corroborarse una tendencia a la «universalización» del gusto grupal: en ambos premios las traducciones realizadas por «traductores veteranos», "profesores universitarios» y otros grupos relacionados con la universidad («traductores-profesores») representan un porcentaje muy superior al resto $(24$ de 32 en el premio a una traducción, I5 de 21 en el premio a una carrera); es decir, la configuración profesional de los jurados tiende a reproducirse en los premiados. De este modo, se observa una vez más que la categoría profesional y el tipo de formación constituyen dos parámetros necesarios para comprender las condiciones sociales del «gusto». En este sentido, la trayectoria de los Premios Nacionales puede interpretarse a partir de la oposición entre dos grupos profesionales 
Tabla io. Premios Nacionales a la Mejor Traducción (1984-2009) Desglosados SEGÚN LA LENGUA DE ORIGEN DE LA OBRA TRADUCIDA Y EL SEXO DEL PREMIADO

\begin{tabular}{lcccc}
\hline & \multicolumn{5}{c}{ Sexo traductor } \\
\hline Lengua origen & $H$ & $M$ & $E q$ & Total \\
\hline Inglés & 3 & 2 & $\circ$ & 5 \\
\hline Latín & 5 & $\circ$ & $\circ$ & 5 \\
\hline Griego clásico & 2 & $\mathrm{I}$ & $\circ$ & 3 \\
\hline Griego moderno & 2 & $\mathrm{I}$ & $\circ$ & 3 \\
\hline Italiano & 3 & $\circ$ & $\circ$ & 3 \\
\hline Portugués & 3 & $\circ$ & $\circ$ & 3 \\
\hline Alemán & 2 & $\circ$ & $\circ$ & 2 \\
\hline Arabe & 2 & $\circ$ & $\circ$ & 2 \\
\hline Chino & 2 & $\circ$ & $\circ$ & 2 \\
\hline Varias & 2 & $\circ$ & $\circ$ & 2 \\
\hline Albanés & $\mathrm{I}$ & $\circ$ & $\circ$ & $\mathrm{I}$ \\
\hline Serbio & $\circ$ & $\circ$ & $\mathrm{I}$ & $\mathrm{I}$ \\
\hline Total & 27 & 4 & $\mathrm{I}$ & 32 \\
\hline
\end{tabular}

- traductores y profesores universitarios (filólogos) — que eligen a partir de las disposiciones y expectativas adquiridas durante su socialización, pero que, igualmente, pueden ejercer su labor de legitimación institucional como una forma de delimitación intelectual, es decir, postulando una tarea intelectual - poco definida a priori- como privativa de un modo concreto de profesionalización: los «filólogos» tienden a preferir las traducciones hechas por «filólogos», los «traductores» aquellas de «traductores», etc. (cf. Fernández, 2orıa).

¿En qué medida pueden estos datos y argumentos reforzar o contradecir lo planteado en el epígrafe anterior acerca de las traductoras? De manera bastante clara, el análisis de los premios parece reforzar el planteamiento de que la «multiposicionalidad» laboral, la «legitimidad» de los
Tabla it. Premios Nacionales a la Obra DE UN Traductor (1989-2009) Desglosados SEGÚN LA CATEGORÍA PROFESIONAL Y EL SEXO DEL PREMIADO

\begin{tabular}{lccc}
\hline & \multicolumn{3}{c}{ Sexo traductor } \\
\hline Estatuto prof. & $H$ & $M$ & Total \\
\hline Traductor veterano & 6 & 3 & 9 \\
\hline Poeta-traductor & 4 & I & 5 \\
\hline Profesor & 4 & 0 & 4 \\
\hline Traductor-profesor & 2 & 0 & 2 \\
\hline Escritor-traductor & I & 0 & I \\
\hline Total & I7 & 4 & $2 \mathrm{2I}$ \\
\hline
\end{tabular}

géneros literarios y la «centralidad» de las lenguas son factores que influyen de manera muy clara en la visibilidad y el reconocimiento social: en el Premio Nacional a la Mejor Traducción (tabla 8), donde la docencia universitaria tiende a constituir una variable importante, las mujeres suponen un bajo porcentaje de premiados (4 premios, $\mathrm{I} 2,5 \%$ del total); al mismo tiempo, en el Premio Nacional a la Obra de un Traductor (tabla II), donde la condición profesional pesa más - por el hecho lógico de que el premio presuponga una trayectoria y continuidad-, la presencia de traductoras no aumenta de modo destacable y siguen siendo minoría (4 premios, 19,5\% del total). Considerando también los géneros y lenguas para ambos premios (tablas 9, IO, I2 y I3), se observa de nuevo la escasa presencia de las traductoras en el género «noble» de la poesía - de especial importancia en la historia del Premio a la Mejor Traducción-y en algunas lenguas de alto capital literario, como las clásicas y el alemán. Puede considerarse, por lo tanto, que los reconocimientos institucionales vuelven a señalar el mismo factor de división 
Tabla i2. Premios Nacionales a la

Obra de un Traductor (1989-2009)

DESGLOSADOS SEGÚN EL GÉNERO LITERARIO

60
DE ESPECIALIZACIÓN Y EL SEXO DEL PREMIADO

Sexo traductor

\begin{tabular}{lccc}
\hline Género & $H$ & $M$ & Total \\
\hline Narrativa & 7 & 3 & Io \\
\hline Poesía & 4 & I & 5 \\
\hline Varios & 4 & $\circ$ & 4 \\
\hline Ensayo & 2 & $\circ$ & 2 \\
\hline Total & I7 & 4 & 2 I \\
\hline
\end{tabular}

entre «traductores» $\mathrm{y}$ «traductoras», aquel que diferencia la (relativa) visibilidad de unos frente a la invisibilidad casi absoluta de otras: la propia configuración del campo editorial y, en consecuencia, el desarrollo (voluntario o condicionado) por parte de los sujetos de un tipo de trayectoria que, orientada hacia unos u otros ámbitos de especialidad, acaba otorgándoles mayor o menor reconocimiento simbólico.

\section{CONCLUSIONES E HIPÓTESIS}

El estudio - sintético, pero de amplia base empírica- de dos ámbitos culturales «legítimos» (crítica literaria y premios institucionales) evidencia que, pese a su mayor presencia y mayor implicación en el sector profesional, las traductoras tienden a ocupar una posición dominada: realizan su tarea allí donde menos visible resulta, ya que traducen obras y géneros menos «formales» y, por tanto, menos legítimos y de menor interés para la crítica; tienen menos presencia en las lenguas centrales y tienden a destacar en lenguas periféricas o semiperiféricas; no suelen compaginar su tarea profesional con otras más prestigiadas del campo literario, como la escritura; y sus trayectorias suelen alcanzar
Tabla i3. Premios Nacionales a la Obra DE UN Traductor (1989-2009) Desglosados SEGÚN LA LENGUA DE ESPECIALIZACIÓN Y EL SEXO DEL PREMIADO

\begin{tabular}{lccc}
\hline & \multicolumn{3}{c}{ Sexo traductor } \\
\hline Lengua & $H$ & $M$ & Total \\
\hline Alemán & 6 & $\circ$ & 6 \\
\hline Varias & 4 & 2 & 6 \\
\hline Griego clásico & 4 & $\circ$ & 4 \\
\hline Inglés & 2 & I & 3 \\
\hline Francés & $\circ$ & I & I \\
\hline Italiano & I & $\circ$ & I \\
\hline Total & I7 & 4 & $2 \mathrm{I}$ \\
\hline
\end{tabular}

un menor reconocimiento institucional. Si bien resulta complejo analizar el conjunto de mecanismos que posibilitan esta dominación masculina, parece factible plantear algunas hipótesis a partir de las connotaciones sociales de los «roles», de la «multiposicionalidad» y de las propias expectativas personales.

En primer lugar, como se ha observado, la mayor implicación en el campo literario, de una parte, y en el universitario, de otra, constituyen factores de importancia para obtener un mayor reconocimiento en la tarea traductora. Ambas profesiones no sólo están connotadas socialmente como «masculinas» — piénsese en la tipología «profesor» vs. «maestra» o, en otro campo muy distinto, «médico» vs. «enfermera» (cf. los datos de Anker, r998: 288)4_ sino que se sitúan en una posición dominante para la «universalización del gusto» que estructura el debate por la legitimidad literaria y traductora;

4 Por otro lado, de acuerdo con datos recientes (Tobarra, 20Io), las mujeres suponen la mayoría del alumnado universitario (55\%), pero su presencia desciende de forma brusca en los puestos de enseñanza, ya que sólo representan el $36 \%$ del profesorado titular y el I $4 \%$ de los catedráticos. 
de esta forma, la multiposicionalidad — de la que carecerían las traductoras profesionalessupondría un factor importante para producir el valor social de la traducción. En esta línea se manifiestan dos traductoras encuestadas:

[...] no sé si los editores se paran a pensar si su traductor es hombre o mujer, lo que sí me parece es que, en general, los hombres que se dedican a la traducción se dan más aires intelectuales (o son verdaderos intelectuales), mientras que las mujeres teorizan menos (siempre hay excepciones, desde luego), y eso puede dar lugar a que un editor se deje impresionar más por un traductor que por una traductora de calidad comparable pero que se dé menos aires. (Traductora I)

Lo de los premios yo lo relaciono más bien con una tendencia general al hecho de que las mujeres en este país no ocupan altos cargos, y el de catedrático es un «alto cargo» dentro de la Academia, y el premio suele concederse a catedráticos o similares (siempre hombres, es cierto) bien por razones políticas o de «prestigio», bien porque no son traductores intensivos sino esporádicos [...]. (Traductora 3 )

En segundo lugar, debe considerarse el modo en que cada grupo se implica laboralmente, es decir, en qué medida uno y otro sexo se interesan por la profesión «en sí misma» o por la profesión «como medio» para acceder a otro beneficio, cuestión que puede estar condicionada también por la tradición y por el carácter social de las expectativas. De acuerdo con un estudio en torno al prestigio laboral y el sexo de los sujetos (Gómez Bueno, I996), las mujeres españolas encuestadas tendían a preferir profesiones feminizadas, ya que consideraban más importantes las características de cada puesto y las posibilidades de acceso que el prestigio asociado a él; los hombres, en cambio, tendían a preferir empleos de mayor prestigio, poniendo en un segundo plano las características y las posibilidades de acceso. En esta línea, un estudio mediante cuestionarios realizado en los Países Bajos (De Jong, I999) señalaba que las traductoras tendían a implicarse más en su trabajo por motivaciones intrinsecas - el trabajo «en sí», el interés o satisfacción que les aporta$\mathrm{ba}$-, mientras que los traductores valoraban más aquellas extrínsecas, es decir, los beneficios que el trabajo les proporcionaba en términos de ganancias o reconocimiento social. Estos planteamientos empíricos coinciden con lo declarado por una de las traductoras entrevistadas durante esta investigación:

Sí... los pocos hombres que se dedican a esto tienen carreras más brillantes que la media. [...] No creo que sea exactamente porque ellas tienen más trabas sino porque ellos lo tienen más claro. Si no ven perspectivas de buenos ingresos o una carrera brillante, lo dejan. Quiero decir, que el nivel de exigencia de las mujeres es menor en todos los sentidos... Porque es una actividad complementaria a otras profesiones, porque necesitan los ingresos desesperadamente... es fácil que la mujer trabaje a destajo sin plantearse demasiadas cosas... En cambio, veo en los varones... más afán de hacer currículum, de ganar el Premio Nacional, de tener una carrera académica... (Traductora I)

En suma, tantos los datos como las declaraciones personales parecen sugerir una tendencia entre las mujeres a valorar la profesión en sí misma y a considerar más importante el trabajo que el prestigio derivado. Las explicaciones tradicionales, aquellas que «naturalizan» lo social, relacionarían esta tendencia con alguna supuesta "característica innata» del sexo femenino (mayor «racionalismo», «sensatez»), encubriendo su origen. Sociológicamente, sin 
embargo, la forma de concebir la profesión debe explicarse a partir de la estructura social y la configuración del mercado: habida cuenta del lento acceso de las mujeres (españolas) al mercado laboral y, sobre todo, de su difícil acceso a las profesiones intelectuales, parece factible que sus expectativas estén más condicionadas por las posibilidades (Bourdieu, I980: I08; 1998: 8o-83) que por los rendimientos asociados y que la gestión de sus carreras se plantee desde criterios distintos a los de sus compañeros varones. En un ámbito artístico semejante, por ejemplo, dos estudios recientes sobre las intérpretes de música clásica en Francia (Ravet, 2003, 2007) mostraban que, a diferencia de los varones, las mujeres tendían a considerar el acceso a la orquesta como un éxito «en sí mismo», dadas las dificultades de selección; asimismo, a la hora de tomar decisiones, su vida familiar (cónyuge e hijos) constituía para ellas un factor de mayor importancia que en el caso de sus compañeros varones. Convendría, por tanto, considerar esta actitud de las mujeres ante la profesión - preferencia por la accesibilidad, motivaciones intrínsecas- a partir de su «habitus», es decir, considerando su trayectoria en un ámbito social caracterizado por la restricción de posibilidades y, por tanto, de expectativas. Puede recordarse aquí un preciso pasaje de las memorias de la editora británica Diana Athill (2000 [2002]: 55-58), donde describe sus dificultades para asentarse en la profesión; a través de un autoanálisis retrospectivo, llega a comprender cómo los condicionantes sociales la llevaron a limitarse: aunque era evidente el favoritismo con el que se trataba a sus compañeros de trabajo varones, nunca se sintió dispuesta a quejarse, ya que «en realidad te veías - al menos en parte- como los hombres te veían, con lo cual sabías qué iba a suceder en caso de ponerte reivindicativa y comportarte de

una forma que a los hombres les resultase ridícula y tediosa. De manera grotesca, tú misma empezarías a parecerte ridícula y tediosa.» (Athill, 2000: 56; cursivas mías).

Asimismo, es pertinente considerar que, allí donde una profesión poco legitimada permite mayores rendimientos - económicos o simbólicos-, tiende a masculinizarse. Así ocurrió, por ejemplo, con el género novelístico durante el siglo XIX (Tuchman, I989): si antes de I840, cuando este género literario poseía escaso prestigio intelectual, las mujeres suponían la mitad de los novelistas en Gran Bretaña, el desarrollo económico del sector editorial y el aumento de beneficios se acompañó pronto por una masculinización de la narrativa. En consecuencia, aunque la traducción haya servido como acceso de las mujeres al sector editorial y que esta tendencia, como demuestran los datos, se siga manteniendo en la actualidad con una feminización de la tarea, la desigualdad sexual no desaparece: en tanto que labor intelectual considerada «inferior», sus rendimientos económicos son bajos y son las mujeres quienes predominan en la profesión; en cambio, en tanto que tarea relacionada con la literatura y la cultura, sus rendimientos simbólicos - premios, becas, obras canónicas - demuestran ser más altos y son los hombres quienes tienden a monopolizarlos.

Estos datos, oposiciones e hipótesis demostrarían que, como ya se ha analizado en numerosos ámbitos profesionales (Maruani \& Nicole, 1989), una mayor implicación profesional de las mujeres no va necesariamente acompañada de un mayor reconocimiento, sino que, por el contrario, suele producir una concentración en las posiciones menos valoradas del sector. De este modo, si los traductores se constituyen como grupo social «dominado» en el ámbito literario y universitario, las 
traductoras formarían actualmente un grupo dominado dentro de los dominados (Kalinowski, 2002: 53).

RECIBIDO EN JULIO DE 2 OII ACEPTADO EN NOVIEMBRE DE 2OII VERSIÓN FINAL DE DICIEMBRE DE 2OII

\section{REFERENCIAS BIBLIOGRÁFICAS}

Agorni, M. (2005). «A Marginal(ized) Perspective on Translation History: Women and Translation in the Eighteenth Century». META, 50/3, pp. $8 \mathrm{i} 7-830$.

Albenga, V. (2007). «Le genre de «la distinction»: la construction réciproque du genre, de la classe et de la légitimité littéraire dans les pratiques collectives de lecture». Sociétés Eं Représentations, 24, pp. 16i-i76.

Anker, R. (1998). Gender and jobs. Sex segregation of occupations in the world. Segunda edición corregida [200I]. Ginebra: International Labour Office.

Athill, D. (200o). Stet: An Editor's Life, Nueva York: Grove Press.

Bielby, D. (2009). "Gender inequality in culture industries: Women and men writers in film and television». Sociologie du travail, 51, pp. 237-252.

Bourdieu, P. (1979). La Distinction. Critique sociale du jugement, París : Éditions de Minuit.

Bourdieu, P. (I980). Le sens pratique, París: Éditions de Minuit.

Bourdieu, P. (1998). La domination masculine, París: Éditions du Seuil.

Buscatto, M. (2007). Femmes du jazz. Musicalités, féminités, marginalisations, París: CNRS Éditions.

Cacouault-Bitaud, M.; Ravet, H. (2008). «Les femmes, les arts et la culture. Frontières artistiques, frontières de genre». Travail, genre et sociétés, I9/I, pp. I9-22.

Chamberlain, L. (I988). «Gender and the Metaphorics of Translation». Signs, I3/3, pp. 454-472.

Cockburn, C. (1987). Two-track training. Sex Inequalities and the YTS, Londres: Macmillan Education.

De Jong, E. (I999). «The impact of motivation on the career commitment of Dutch literary translators». Poetics, 26, pp. 423-437.
Delisle, J. (2002). Portraits de traductrices, Ottawa: Presses de l'Université d'Ottawa.

Fernández, F. (2010). "La traducción literaria y la brecha de paralaje. Reflexiones a partir de un cuestionario piloto». MONTI. Monográficos en Traducción e Interpretación, 2, pp. 193-215.

Fernández, F. (20Ira). «Las condiciones sociales del juicio. Aportaciones al estudio de la recepción crítica de literatura traducida». Quaderns. Revista de traducció, 18: 187-198.

Fernández, F. (20IIb). La recepción crítica de literatura traducida en España (1999-2008): aportaciones a una sociología de la literatura transnacional, Universidad de Granada: Tesis doctoral inédita.

Gómez Bueno, C. (I996). «El género y el prestigio profesional». REIS, 75, pp. 215-233.

Heilbron, J. (I999). «Toward a Sociology of Translation: Book Translations as a Cultural WorldSystem». European Journal of Social Theory, 2/4, pp. 429-444.

Heilbron, J. (2000). «Translation as a cultural world system». Perspectives, 8/ı, pp. 9- 26.

Ibáñez Pascual, M. (2008). «La segregación ocupacional por sexo a examen: características personales, de los puestos y de las empresas asociadas a las ocupaciones masculinas y femeninas». REIS, I23, pp. 87-I22.

Ibáñez Pascual, M. (20ıo). «Al otro lado de la segregación ocupacional por sexo. Hombres en ocupaciones femeninas y mujeres en ocupaciones masculinas». Revista Internacional de Sociologia, 68/I, pp. I45-164.

Kalinowski, I. (2002). "La vocation au travail de traduction». Actes de la recherche en sciences sociales, I44, pp. 47-54.

Kalinowski, I. (2007). «Denise Naville traductrice» en Blum F. (ed), Les vies de Pierre Naville, Lille: Presses du Septentrion, pp. $5 \mathrm{I}^{-} 63$.

Kant, I. (I764)/(I978). Beobachtungen über das Gefübl des Schönen und Erhabenen, Riga: J.F. Hartknoch; trad. esp. de Manuel García Morente. Prolegómenos a toda Metafísica del Porvenir. Observaciones sobre el sentimiento de lo bello y lo sublime. Crítica del Juicio. México: Porrúa.

Macías Sistiaga, C.; Fernández-Cid, M. (2003). «Informe sobre la situación del traductor de libros en España». [En línea] ACE traductores, www. acett.org/documentos/informe_completo_definitivo. $p d f$ [Consulta: Io de julio de 2007]. 
Macías Sistiaga, C.; Fernández-Cid, M.; Martín Caño, Á. (I997). «Encuesta a traductores. Informe de resultados» en ACE Traductores (eds.), Libro Blanco de la Traducción en España, Madrid: ACE Traductores, pp. 25-95.

64
Marinas, J. M. (20I0). «Estudio» en ACE Traductores (eds.), Libro Blanco de la Traducción, Madrid: Ministerio de Cultura \& ACE Traductores, pp. 37-83.

Maruani, M.; Nicole, C. (1989). Au labeur des dames. Métiers masculins, emplois féminins, París: Syros.

Matter-Seibel, S. (2006). «Margaret Fullers Übersetzungen deutscher Werke : Soziale Entstehungsbedingungen und genderspezifische Aspekte» en Wolf M (ed.), Übersetzen - Translating - Traduire: Towards a 'Social Turn'?, Münster \& Viena: LIT Verlag, pp. 23-33.

Naudier, D. (2007). «Les modes d'accès des femmes écrivains au champ littéraire contemporain» en Mauger G. (dir.), Droits d'entrée. Modalités et conditions d'accès aux univers artistiques, París: Éditions de la Maison des sciences de l'homme, pp. I9I-2I3.

Naudier, D. (2010). "Les écrivaines et leurs arrangements avec les assignations sexuées». Sociétés contemporaines, 78, pp. 39-63.

Papí, N.; Frau, M. J. (2005). «La conciliación del empleo y del hogar: respuesta y reflejo de una organización del trabajo construida desde la institución del género». REIS, ıı, pp. I49-I7I.

Pieretti, M.-P. (2002). «Women Writers and Translation in Eighteenth-Century France». The French Review, 75/3, pp. 474-488.

Ravet, H. (2003). «Professionnalisation féminine et féminisation d'une profession: les artistes interprètes de musique». Travail, genre et sociétés, 9, pp. I73-195.

Ravet, H. (2007). «Devenir clarinettiste. Carrières féminines en milieu masculin». Actes de la recherche en sciences sociales, 168 , pp. 50-67.

Sirois, A. (1997). Les femmes dans l'bistoire de la traduction : de la Renaissance au XIXe siècle : domaine français, Université d'Ottawa: Tesis doctoral inédita.

Tobarra, S. (2010). «Más formadas, pero con peores empleos». El País, I4 de noviembre de 20Io, pp. 36-37.

Trasforini, M.-A. (2007). "Elles deviendront des peintres'. Femmes artistes et champ social de l'art» en Lachat S. \& Fidecaro A. (dir.), Profession: créatrice. La place des femmes dans le champ artistique, Lausana: Antipodes, pp. 25-47.

Tuchman, G. (1989). Edging Women Out: Victorian Novelists, Publishers, and Social Change, New Haven: Yale University Press. 